\title{
Rechtsprechung
}

\section{Anspruch auf Löschung eines Eintrages in der Lost Art Datenbank}

\author{
Oberverwaltungsgericht Sachsen-Anhalt, Urteil vom 23. Oktober 2013 - 3 L 84/12
}

1. Der Anspruch auf Löschung eines Eintrages in der Lost Art Datenbank bestimmt sich nach den für die Rechtmäßigkeit staatlichen Informationshandelns entwickelten Grundsätzen.

2. Eine Eintragung in der Suchliste der Lost Art Datenbank hat ihren Zweck erfüllt, wenn der als verschollen gemeldete Kunstgegenstand aufgefunden worden ist und dies den Personen, welche Eigentums-, Besitz- oder Restitutionsansprüche an dem Kunstwerk geltend machen, bekannt ist. Die Suchliste hat nicht den Zweck, Ansprüche verschiedener Eigentumsprätendenten zu sichern. (Amtliche Leitsätze)

\section{Gründe}

\section{I.}

- Die Klägerin begehrt vom Beklagten die Löschung einer Suchmeldung, welche in der vom Beklagten im Internet geführten Lost Art Datenbank eingetragen ist. Dem Beklagten ist als Arbeitsgruppe die Koordinierungsstelle A-Stadt angeschlossen, welche eine von der Bundesrepublik Deutschland und den Bundesländern finanzierte Einrichtung darstellt, die u.a. die Aufgabe hat, "Such- und Fundmeldungen des In- und Auslands zu NS-verfolgungsbedingt entzogenen bzw. in Folge des 2. Weltkriegs verbrachten Kulturgütern zur Präsentation in www.lostart.de" zu dokumentieren.

Unter dem 24. Juni 2005 erfolgte im Auftrag der Erbengemeinschaft nach R. und J. O. die Eintragung einer Suchmeldung in der Lost Art Datenbank hinsichtlich des hier in Rede stehenden Gemäldes „Bildnis eines alten Mannes in orientalischer Tracht", welches ursprünglich Rembrandt Harmenszoon van Rijn zugeschrieben wurde und heute Isaac Jouderville, einem Schüler Rembrandts, zugeordnet wird.

Dem liegt im Wesentlichen folgender Sachverhalt zugrunde: Im Jahr 1912 gründete Albert L. die Margraf \& Co. GmbH in C-Stadt. In den folgenden Jahren wurde diese Gesellschaft um mehrere Untergesellschaften erweitert, namentlich die Kunsthandelsgesellschaften F. \& Co. GmbH, Dr. Benedict \& Co. GmbH, Dr. Burchard \& Co. GmbH sowie die Antiquitätenhandelsgesellschaft Altkunst \& Co. GmbH. Albert L. legte die Geschäftsführung hinsichtlich dieser Unternehmensgruppe in die Hände des Kunsthändlers J. O. und dessen Ehefrau R., welche wie Albert L. beide jüdischen Glaubens waren. Vor seinem Tod im Jahr 1929 hatte Albert L. seine Lebensgefährtin R. B. als Erbin eingesetzt und die Anteile an den vorgenannten Gesellschaften als Vermächtnis den Eheleuten O. hinterlassen. Diese Rechtsnachfolge hinsichtlich der Gesellschaften wurde jedoch - nach den vorliegenden Akten - aufgrund von erbrechtlichen Auseinandersetzungen bis 1933 nicht registerrechtlich vollzogen. Am 1. April 1933, versuchten die Nationalsozialisten die Eheleute O. zu verhaften. Diese waren jedoch gewarnt worden und konnten nach
Frankreich fliehen. J. O. verstarb 1941 in Nizza. R. O. wurde nach Auschwitz deportiert und dort 1943 ermordet. Die drei Kinder der Eheleute O. überlebten die Zeit des Nationalsozialismus. Die Gesellschaftsanteile der vorgenannten Unternehmensgruppe wurden nach 1933 zugunsten des Finanzamtes Berlin-Tiergarten für ausstehende Erbschaftssteuern verpfändet. Als die Steuerschulden 1937 beglichen wurden, wurden diese Gesellschaftsanteile an R. B. übertragen. Bereits unter dem 2. Dezember 1933 hatte das Landgericht Berlin J. O. untersagt, jedwede Rechtshandlungen in Bezug auf die Unternehmensgruppe vorzunehmen. Prof. Dr. Bolko Freiherr von Richthofen, nach den vorliegenden Unterlagen ein enger Freund Hermann Görings, wurde im Jahr 1933 zum Verwalter der Unternehmen bestimmt. Etwa ab dem Jahr 1935 wurden die Untergesellschaften der Unternehmensgruppe liquidiert. In einem Auktionstermin (26. und 27. April 1935), der - laut Katalog - die „Bestände der Berliner Firmen Galerie F. \& Co. GmbH und Altkunst Antiquitäten $\mathrm{GmbH}^{\text {“ betraf }}$ wurde auch das streitgegenständliche Gemälde vom Auktionshaus G. in C-Stadt angeboten und für 16.000 RM versteigert. Es soll - nach dem Vortrag der Beigeladenen - vom Bankhaus Jacquier \& Securius mit Sitz in C-Stadt ersteigert worden sein. Zum Beleg verweisen die Beigeladenen auf einen Auszug aus der "Liste der national wertvollen Kunstwerke" von 1938.

Die Beigeladenen sind Mitglieder von Erbengemeinschaften, die die (jüdischen) Gesellschafter des ehemaligen Bankhauses Jacquier \& Securius beerbt haben. Unter dem 10. September 2009 erwirkten die Mitglieder der Erbengemeinschaften die Registrierung einer (weiteren) Suchmeldung hinsichtlich des streitgegenständlichen Gemäldes bei dem Beklagten. Zur Begründung dieser Suchmeldung haben die Beigeladenen vorgetragen, dass sich das Gemälde seit Oktober 1933 im Sicherungseigentum des Bankhauses befunden habe, dieses Sicherungseigentum sei im Rahmen der Versteigerung im Jahr 1935 zu Volleigentum erstarkt und erst durch die sogenannte Arisierung des Bankhauses im März 1938 verfolgungsbedingt abhanden gekommen.

Im Mai 2009 gelang es der Nachtragsliquidatorin der Klägerin unter Hinweis auf die Eintragung der Suchmeldung unter anderem in der Lost Art Datenbank, eine unmittelbar bevorste- 
hende Versteigerung des streitgegenständlichen Gemäldes auf einer Auktion in Kapstadt zu verhindern. Das Bild war von dem in Windhoek/Namibia lebenden Gerhard-Peter S. angeboten worden.

Im Januar 2010 schlossen Gerhard-Peter S., die Klägerin und die Erbengemeinschaft nach den vormaligen Gesellschaftern der Galerie F. \& Co. GmbH eine Vereinbarung über die Verwertung des hier in Rede stehenden Gemäldes. Es soll bei dem Auktionshaus Sotheby's in Amsterdam versteigert und der Erlös hälftig zwischen Herrn Gerhard-Peter S. und der Erbengemeinschaft nach J. und R. O. geteilt werden. Zu dieser Versteigerung ist es noch nicht gekommen.

Der Beklagte lehnte die nachfolgend von der Klägerin geforderte Löschung der Suchmeldung mit der Begründung ab, dass eine - für plausibel erkannte - Eintragung nur mit Zustimmung des (weiteren) Melders - hier der Beigeladenen - gelöscht werden dürfe. Wenn nötig, müsse die Klägerin die Zustimmung der Beigeladenen auf dem Zivilrechtsweg erstreiten.

Am 24. Juni 2010 hatte die Klägerin Klage gegen das Land Sachsen-Anhalt, vertreten durch das Kultusministerium, dieses vertreten durch die Koordinierungsstelle A-Stadt, erhoben. Zur Begründung hat sie im Wesentlichen ausgeführt: Die allgemeine Leistungsklage sei die statthafte Klageart, da die Eintragung auf der Internetseite www.lostart.de ein schlichtes Verwaltungshandeln darstelle. Der Beklagte sei passiv legitimiert. Da er die Eintragung der Suchmeldung vorgenommen und zu verantworten habe, müsse er sie auch löschen können. Der Klägerin stehe ein Folgenbeseitigungsanspruch zu. Der Verbleib der Suchmeldung in der Datenbank beeinträchtige das Eigentumsrecht der Klägerin, welche als Erstgeschädigte das bessere Recht habe.

\section{Die Klägerin hat beantragt,}

den Beklagten zu verurteilen, den (von den Beigeladenen veranlassten) Eintrag des Gemäldes „Bildnis eines alten Mannes in orientalischer Tracht" von Rembrandt (mittlerweile Jouderville, Schüler Rembrandts, zugeschrieben) von der Liste gesuchter Raub-und Beutekunst auf der Internetseite www.lostart.de zu löschen,

hilfsweise, den Beklagten zu verurteilen, der Klägerin Auskunft über die Namen und Anschriften der Personen oder Institutionen zu erteilen, die den Eintrag des Gemäldes „Bildnis eines alten Mannes in orientalischer Tracht" von Rembrandt (mittlerweile Jouderville, Schüler Rembrandts, zugeschrieben) auf der Liste gesuchter Raub- und Beutekunst auf der Internetseite www.lostart.de veranlasst haben. Sofern ein Vertreter, beispielsweise ein Rechtsanwalt die Eintragung veranlasst hat, sind auch die von ihm vertretenen Personen und Institutionen mit Namen und Anschrift zu benennen.

Der Beklagte hat beantragt,

die Klage abzuweisen.
Zur Begründung hat er ausgeführt: Die Klage sei unzulässig, weil die Klägerin den Beklagten falsch bezeichnet habe. Sie habe auch kein Rechtschutzbedürfnis, weil sie mit einer Klage auf Zustimmung zur Löschung des Eintrags vor den Zivilgerichten leichter und schneller zum Ziel komme. Zudem sei die Klage unbegründet. Der Beklagte dürfe die Eintragung zugunsten der Beigeladenen nicht ohne Zustimmung der anmeldenden Personen löschen und zurücknehmen. Der Klägerin stehe ein Folgenbeseitigungsanspruch nicht zu. Der Beklagte beeinträchtige das Eigentum der Klägerin nicht. Die Eintragung bewirke kein rechtliches Verfügungsverbot. Dass die Auktionshäuser die Internetseite www.lostart.de beachten, liege in deren Verantwortungsbereich. Ohne positive Feststellung des Eigentums der Klägerin und/oder ohne Zustimmung der Beigeladenen könne der in Rede stehende Eintrag nicht gelöscht werden.

Die Beigeladenen hatten keinen Antrag gestellt. Sie haben geltend gemacht, die Klägerin behaupte zu Unrecht, Eigentümerin des in Rede stehenden Gemäldes zu sein. Das Bild könne auch einer namensgleichen Galerie in Amsterdam oder einem Dritten gehört haben. Selbst wenn die Klägerin die ursprüngliche Eigentümerin des Gemäldes gewesen wäre, hätte sie das Eigentum spätestens durch die Sicherungsübereignung an das Bankhaus Jacquier \& Securius vom 13. Oktober 1933 verloren. Die Sicherungsübereignung und die spätere Verwertung seien weder rechtlich noch sittlich zu beanstanden. Mit der Sicherungsübereignung seien verfolgungsunabhängige Kredite besichert worden. Der Erbschaftsstreit, die Erbschaftssteuerschulden und die Weltwirtschaftskrise hätten die Unternehmensgruppe, zu welcher auch die F. \& Co. GmbH gehört habe, stark belastet. Auch aus steuerlichen Gründen seien J. und R. O. ins Ausland gegangen bzw. im Ausland geblieben. Einen verfolgungsbedingten Kulturgutverlust habe hingegen das Bankhaus Jacquier \& Securius erlitten. Im Oktober 1933 habe es Sicherungseigentum unter anderem an dem streitgegenständlichen Gemälde erworben. Im April 1935 habe das Bankhaus das Gemälde ersteigert und auf diese Weise Volleigentum erlangt. Die „Liste der national wertvollen Kunstwerke 1938“ belege den Erwerb des Gemäldes durch das Bankhaus Jacquier und Securius. Zum 1. März 1938 sei das Bankhaus „arisiert“ worden. Das sei die einzige verfolgungsbedingte Schädigung in Ansehung des hier in Rede stehenden Gemäldes.

Mit Urteil vom 17. Januar 2012 hat das Verwaltungsgericht das Kultusministerium des Landes Sachsen-Anhalt verurteilt, den streitgegenständlichen Eintrag in der Lost Art Datenbank zu löschen. Zur Begründung hat es ausgeführt: Der Rechtsweg zu den Verwaltungsgerichten sei eröffnet. Das zwischen der Klägerin und dem Beklagten bestehende Rechtsverhältnis sei dem öffentlichen Recht zuzuordnen, weil das Klageziel, die Löschung des Interneteintrags, vom staatlichen Wiedergutmachungsauftrag abhänge, der in der Vereinbarung der Bundesrepublik Deutschland mit den Bundesländern und den kommunalen Spitzenverbänden einerseits und in den sogenannten Washingtoner Grundsätzen von 1998 andererseits seinen Ausdruck gefunden habe. Der Streit um Einträge auf dieser Internetseite sei 
- wegen des beabsichtigten Zusammenhangs mit dem staatlichen Wiedergutmachungsauftrag - eine öffentlich-rechtliche Streitigkeit. Die Klägerin müsse sich zur Verfolgung ihres Begehrens nicht auf den Zivilrechtsweg verweisen lassen. Eine Eigentumsfeststellungsklage oder eine gegen die Beigeladenen gerichtete Leistungsklage auf Erteilung der Zustimmung zur Löschung des Eintrags auf der Internetseite würde nicht einfacher, kostengünstiger oder schneller zum angestrebten Erfolg führen, zumal (noch) nicht alle Rechtsnachfolger der Inhaber des ehemaligen Bankhauses Jacquier \& Securius benannt worden seien. Eine - relevante - Falschbezeichnung des Beklagten im Sinne des § 78 Abs. 1 VwGO in Verbindung mit § 8 AGVwGO LSA liege nicht vor. Die zulässige allgemeine Leistungsklage sei auch begründet. Die Klägerin habe einen Anspruch auf Löschung der Suchmeldung hinsichtlich des streitgegenständlichen Gemäldes, weil - was der Beklagte beachten müsse - die Klägerin Erstgeschädigte sei und - was selbstständig tragend hinzu komme - Zweckerreichung eingetreten sei. Zwar enthielten weder die Washingtoner Grundsätze von 1998 noch die "Gemeinsame Vereinbarung über die Koordinierungsstelle A-Stadt“ vom 9. Februar 2010, die die Bundesrepublik Deutschland und die Bundesländer getroffen hätten, subjektive Rechte zugunsten der Klägerin. Wie die Koordinierungsstelle A-Stadt die ihr zugewiesenen Aufgaben erfülle, regele insbesondere die Gemeinsame Vereinbarung nicht. Ebenso wenig sei das Außenrecht der Koordinierungsstelle dort fixiert. Das Fehlen von Bestimmungen vertraglicher oder gesetzlicher Art bedeute aber nicht, dass die „Nutzer", die Klägerin oder die Beigeladenen der Koordinierungsstelle A-Stadt, "rechtsschutzlos ausgeliefert“ wären. Vielmehr führe das Fehlen von Vorschriften dazu, dass allgemeines Verwaltungsrecht Anwendung finde. In diesem Sinne gehörten die Internetseite www.lostart.de und die Koordinierungsstelle A-Stadt zu den öffentlichen Sachen und Einrichtungen, die dazu bestimmt seien, der Allgemeinheit im Rahmen ihres Widmungszwecks zur Verfügung zu stehen. Der Widmungsakt sei in der "Gemeinsamen Vereinbarung" vom 9. Februar 2010 zu sehen, der festlege, dass die gemeinsame Einrichtung des Bundes und der Länder, die Koordinierungsstelle A-Stadt, ihre Arbeit fortsetze, um verfolgungsbedingte Kulturgutverluste zu dokumentieren und den Betroffenen die Chance auf eine „faire und gerechte Lösung" zu eröffnen. In Anwendung dieser Grundsätze habe die Klägerin einen Anspruch auf Löschung der von dem Beklagten zu verantwortenden Suchmeldung, weil sich dieser Eintrag nicht mehr mit dem Auftrag der Koordinierungsstelle vereinbaren lasse. Er sei nicht mehr erforderlich, weil allen Beteiligten bekannt sei, was gesucht werde, wo es sich befinde, wer es habe und wer es suche. Der Zweck der Eintragung habe sich erfüllt. Die Aufrechterhaltung der beanstandeten Eintragung führe auch zu einer Behinderung des Rechtsverkehrs. Im Übrigen unterscheide das Wiedergutmachungsrecht zwischen dem Erst- und Zweitgeschädigten und zwinge den Beklagten, eine für "plausibel“ gehaltene Erstschädigung nicht durch die Aufnahme einer für "plausibel“ gehaltenen Zweitoder Drittschädigung zu entwerten. Die Klägerin habe auch einen Anspruch auf Löschung der Suchmeldung, weil sie berechtigte Anmelderin im Sinne der Washingtoner Grundsätze sei. Sie habe als Erste das Eigentum an dem in Rede stehenden Gemälde verfolgungsbedingt verloren. Sie sei Eigentümerin des Gemäldes gewesen. Dies sei - die Sicherungsübereignung vom 13. Oktober 1933 einmal außer Acht gelassen - durch den Versteigerungskatalog des Auktionshauses G. und durch die eidesstattliche Versicherung eines langjährigen wissenschaftlichen Mitarbeiters der Galerie im Verfahren nach dem Bundesentschädigungsgesetz zur Überzeugung der Kammer belegt. Die Klägerin habe - aufgrund des öffentlich-rechtlichen Benutzungs- und Sachenrechts - einen Anspruch gegen den Beklagten auf Löschung der Meldung, weil in Ansehung des in Rede stehenden Gemäldes alle Meldungen obsolet geworden sind und die Klägerin Erstgeschädigte sei. Ob der Klägerin derselbe Anspruch auch aufgrund eines öffentlich-rechtlichen Abwehranspruchs zustehe, könne offen bleiben.

Mit der auf Antrag des Beklagten und der Beigeladenen mit Beschluss des Senates vom 16. Mai 2013 zugelassenen Berufung trägt der Beklagte vor: Die Klage sei unzulässig, hilfsweise unbegründet. Entgegen der Auffassung des Verwaltungsgerichts sei der Eintrag der Suchmeldung hinsichtlich des streitgegenständlichen Gemäldes in die Lost Art Datenbank nicht justiziabel. Es handele sich um einen justizfreien Akt. Es handele sich bei der Eintragung in die Liste um eine Tätigkeit im fachlich-informativen und deskriptiven Rahmen und mangels inhaltlicher Vorgaben um fachspezifische Tätigkeiten mit Elementen der Ausübung politischen Ermessens auf ministerieller Ebene und mithin bei der Eintragung in der Datenbank um einen justizfreien, nicht öffentlich-rechtlichen Akt. Auch unter dem Aspekt des staatlichen Informationshandelns ergebe sich keine andere Beurteilung der Rechtslage. Es sei von zentraler Bedeutung, dass die Koordinierungsstelle nur deskriptiv tätig sei und ihre nach außen gegebenen Informationen keine eigenen Wertungen enthielten. Eine tatsächliche oder rechtliche Tiefenprüfung - etwa zur Echtheit oder zur Provenienz des Objektes bzw. zur Berechtigung des Melders seitens der Koordinierungsstelle - sehe deren Mandat nicht vor. Es handele sich bei ihrer Tätigkeit um einen mediativ-dialogischen Kommunikationsprozess zur Vervollständigung der Datensammlung. Ferner liege auch eine relevante Falschbezeichnung des Beklagten vor. Das Land Sachsen-Anhalt bzw. das Kultusministerium seien in dem Rechtsstreit nicht passiv legitimiert. Die Klage sei gegen die Koordinierungsstelle zu richten. Ferner sei die Klage auch unbegründet. Ein Folgenbeseitigungsanspruch bestehe nicht, weil kein rechtswidriger Eingriff in das Eigentum der Klägerin im Sinne von Art. 14 GG vorliege. Die Klägerin habe das Eigentum an dem streitgegenständlichen Gemälde nicht hinreichend dargetan. Sie treffe jedoch eine Obliegenheit zum Nachweis. Auch sei keine andere dem Grundrecht aus Art. 14 GG unterfallende Rechtsposition ersichtlich, die die Klägerin innehätte. Bloße Gewinnerwartungen seien von Art. 14 GG nicht geschützt. Es sei zudem völlig unbewiesen, dass kein Auktionshaus oder Galerie ein Werk veräußere, welches in der Lost Art Datenbank verzeichnet sei. Außerdem sei es der Beklagten nicht zurechenbar, ob die Eintragung in die Liste bestimmte, zudem lediglich 
faktische Wirkungen auslöse. Die Verantwortung für die inhaltliche Richtigkeit einer Suchmeldung liege ausschließlich beim Melder.

Die Beigeladenen tragen zur Begründung der Berufung vor, dass es nicht nur Zweck der Lost Art Datenbank sei festzustellen, wer das im Eintrag erwähnte Bild habe, wo es sich befinde und wer es suche. Ein weiterer Zweck des Registers bestehe vielmehr darin, Kunstverluste, die auf NS-Verfolgung zurückgehen können, zu dokumentieren. Die von der Klägerin begehrte Löschung lasse sich mit diesem Zweck nicht in Einklang bringen. Ferner liege entgegen der Auffassung des Verwaltungsgerichts keine Erstschädigung der Klägerin vor. Es sei keineswegs geklärt, dass der Klägerin das Bild jemals gehört habe bzw. dass sie das Bild wegen einer NS-Verfolgung verloren habe. Insbesondere würde die Vermutung für eine Ursächlichkeit der NS-Verfolgung widerlegt werden können. Die Klägerin hätte nämlich in diesem Fall einen wenn auch möglicherweise niedrigeren "Rembrandt-Preis“ für ein Werk erhalten, das nur eine Rembrandt-Fälschung gewesen sei. Der objektive Kaufpreis sei also zu hoch gewesen. Die Klägerin habe durch den Verlust keinen Schaden genommen, sondern daraus Nutzen gezogen. Im Übrigen liege auch kein Eingriff in ein Recht bzw. Interesse der Klägerin vor. Es sei nämlich nicht jedes staatliche Informationshandeln als ein Grundrechtseingriff zu werten. An einem solchen Grundrechtseingriff fehle es schon deshalb, weil der Eintrag keine Publikumsinformation des Beklagten sei. Dies ergebe sich aus dem Inhalt des streitbefangenen Eintrages. Die Lost Art Datenbank sei nur ein Weg von vielen, um dem Markt mitzuteilen, dass jemand hinsichtlich des Werkes ein berechtigtes Wiedergutmachungsinteresse zu haben glaube. Dies werde auch daraus ersichtlich, dass die Eintragung keine Erklärung der Behörde enthalte, sondern dass die Behörde nur eine fremde private Erklärung wiedergebe. Die Beklagte führe kein Register staatlich geprüfter Fälle von Kunstrestitutionsansprüchen. Die Funktion der Lost Art Datenbank sei eher mit der des nichtamtlichen Teils eines Amtsblattes oder eines "schwarzen Brettes" in einem Behördengebäude vergleichbar. Die Erklärung der Beigeladenen, welche die Beklagte veröffentlicht habe, führe nicht zur Verkehrsunfähigkeit des Werkes, sondern nur zu einem Wertverlust. Schon das spreche gegen die Annahme eines Eingriffs. Selbst wenn man einen Eingriff des Beklagten in ein subjektives Recht der Klägerin unterstelle, stimme es mit den Grundsätzen der Rechtmäßigkeit staatlichen Informationshandelns überein, wenn der Beklagte zutreffende Informationen publiziere. Die Löschung der Eintragung würde dem Rechtsverkehr signalisieren, dass niemand mehr Wiedergutmachungsinteressen bezüglich des streitgegenständlichen Werkes verfolge. Dies wäre unzutreffend.

\section{Der Beklagte und die Beigeladenen beantragen,}

das Urteil des Verwaltungsgerichts Magdeburg - 7. Kammer - vom 17. Januar 2012 abzuändern und die Klage abzuweisen.

Die Klägerin beantragt, die Berufungen zurückzuweisen.

Zur Begründung führt die Klägerin im Wesentlichen aus, dass die Klage entgegen der Auffassung des Beklagten und der Beigeladenen zulässig sei. Die Aufnahme von Anträgen in die Lost Art Datenbank führe in verschiedener Hinsicht zu intensiven Eingriffen in die von der Rechtsordnung geschützten Rechte des Eigentümers und/oder Besitzers des jeweiligen Kunstgegenstandes. Der Beklagte habe selbst vorgetragen, dass die Eintragung eines Kunstwerkes in der Datenbank zur faktischen Unverkäuflichkeit eines Bildes führe. Die Tätigkeit des Beklagten sei deshalb rechtserheblich und liege nicht im rechtsfreien Raum. Im Übrigen habe das Verwaltungsgericht auch zutreffend festgestellt, dass keine relevante Falschbezeichnung des Beklagten vorliege. Bei der Koordinierungsstelle A-Stadt handele es sich um eine rechtlich unselbständige Organisationseinheit des Kultusministeriums des Landes Sachsen-Anhalt. Die Klage sei auch begründet. Die rechtswidrige Zweitanmeldung der Beigeladenen verletze die subjektiven Rechte der Klägerin, da sie entgegen der Auffassung der Beigeladenen weiterhin Eigentümerin des streitgegenständlichen Gemäldes sei. Bei den Tätigkeiten der Koordinierungsstelle handele es sich um ein staatliches Informationshandeln. Die Eintragungen in der Lost Art Datenbank seien informatorischer Natur und damit staatliches Informationshandeln, welches sich folglich an Verfassung und Gesetz messen lassen müsse. Mit dem staatlichen Informationshandeln der Koordinierungsstelle könnten Eingriffe in die Grundrechte und weitere subjektive Rechtspositionen des Berechtigten einhergehen. Der Beklagte habe erstinstanzlich selbst vorgetragen, dass die Eintragung eines Kunstwerkes in die Datenbank zur faktischen Unverkäuflichkeit führe. Dies entspreche auch den praktischen Erfahrungen in anderen Fällen. Damit komme der Eintragung die Wirkung eines faktischen Veräußerungsverbotes gleich. Soweit der Beklagte auf einen Fall hinweise, in dem ein Auktionshaus ein Objekt trotz seiner Eintragung im Lost Art Datenbank versteigert habe, handele es sich um einen absoluten Einzelfall, in dem anders als üblich vor der Auktion nicht geprüft worden sei, ob das Kunstwerk als vermisst gelte. Ferner liege der Sinn und Zweck der Lost Art Datenbank in der Identifizierung von Beutekunst und Berechtigten. Die Dokumentation diene dazu, verfolgungsbedingte Kulturgutverluste zu dokumentieren, um den Betroffenen die Chance auf eine faire und gerechte Lösung zu eröffnen. Zweck der Datenbank sei es nicht zu dokumentieren, welche Kunstobjekte eine NS-Raubkunst- oder Beutekunsthistorie aufwiesen. Die Dokumentation habe einzig die Identifikation und das anschließende Zusammenführen von Suchenden und Findenden zum Ziel. Dieses Ziel werde erreicht, wenn die Identitäten von Kunstobjekt, Suchenden und Findenden feststünden, sodass sich die Beteiligten untereinander den Fragen der Restitution widmen könnten. Die Koordinierungsstelle habe nicht die weitergehende Aufgabe, die Restitution zwischen interessierten Personen zu regeln oder zu begleiten. Die Koordinierungsstelle sei vielmehr nach ihren Grundsätzen verpflichtet, die Lost Art Datenbank laufend zu aktualisieren und dabei solche Eintragungen zu löschen, deren Zweck sich erfüllt habe. Das Gebot 
der Aktualisierung und Löschung gelte umso mehr, wenn mit der Eintragung - wie hier - Beeinträchtigungen wegen subjektiver Rechte Dritter, darunter auch die unionsrechtlich geschützte Warenverkehrsfreiheit, Hand in Hand gingen. Die Koordinierungsstelle müsse eine Eintragung daher löschen, wenn sie sich erledigt habe.

\section{II.}

Die Berufungen des Beklagten und der Beigeladenen sind zulässig, jedoch nicht begründet. Das Verwaltungsgericht hat den Beklagten zu Recht verpflichtet, die Suchmeldung hinsichtlich des streitgegenständlichen Gemäldes in der Lost Art Datenbank im Internet zu löschen.

Entgegen der Auffassung des Beklagten ist der Rechtsweg zu den Verwaltungsgerichten gemäß § 40 Abs. 1 VwGO eröffnet. Die Zulässigkeit des Verwaltungsrechtsweges ist auch noch in der Berufungsinstanz zu prüfen, da die Bindungswirkung des $\S$ 17a Abs. 5 GVG nicht eintritt, wenn das erstinstanzliche Gericht das in § 17a GVG vorgesehene (Vorab-)Beschlussverfahren über den zulässigen Rechtsweg nicht beachtet hat und den Rechtsweg zu den Verwaltungsgerichten trotz erstinstanzlicher Rüge erst im Urteil bejaht hat und der betroffene Beteiligte - wie hier der Beklagte - die Rüge der Unzuständigkeit im Berufungsrechtszug aufrechterhält (vgl. OVG Lüneburg, Urteil v. 3. Juli 2001 - 12 LB 955/01 -, [...] mwN). Bei den in Rede stehenden von der Koordinierungsstelle A-Stadt vorgenommenen Eintragungen in die im Internet betriebene Lost Art Datenbank handelt es sich nicht, wie der Beklagte meint, um ein justizfreies staatliches Handeln, welches einer gerichtlichen Kontrolle generell und insbesondere der Kontrolle durch die Verwaltungsgerichte entzogen ist. Der Umstand, dass die Aufgabenerfüllung der Koordinierungsstelle nicht durch Rechtssatz geregelt ist und insbesondere das Rechtsverhältnis zwischen der Koordinierungsstelle und den Nutzern nicht gesetzlich bestimmt ist, indiziert nicht ein solches Reservat nicht gerichtlich überprüfbaren staatlichen Handelns. Ausgangspunkt ist Art. 19 Abs. 4 Satz 1 GG. Nach dieser Vorschrift steht dem, der durch die öffentliche Gewalt in seinen Rechten verletzt wird, der Rechtsweg offen. Diese Norm enthält ein Grundrecht auf effektiven und möglichst lückenlosen richterlichen Rechtsschutz gegen Akte der öffentlichen Gewalt (vgl. BVerfG, Urteil v. 18. Juli 2005 - 2 BvR 2236/04, [...] mwN). Der Umstand, dass die Einrichtung und der Betrieb der Datenbank ggf. nicht der Umsetzung rechtlicher bindender Verpflichtungen dient, berührt nicht die Zulässigkeit der gerichtlichen Kontrolle, sondern unter Umständen nur deren Umfang. Die Entscheidung über die Löschung von Einträgen in der Datenbank ist auch nicht im Kernbereich des Regierungshandelns in Gestalt staatsleitender Hoheitsakte angesiedelt, die sich außerhalb der rechtlich geregelten öffentlichen Lebensbereiche im Gebiet der verantwortlichen politischen Leitung vollziehen und so ihrer Struktur und besonderen politischen Funktion nach unter keinem Gesichtspunkt subjektiv öffentliche Rechte berühren können (vgl. OVG Berlin, Beschluss v. 26. März 2001 - 2 S 2.01, [...] zum Rechtsschutz gegen eine Auslieferungsbewilligung).
Das Verwaltungsgericht hat zutreffend auch eine öffentlichrechtliche Rechtsstreitigkeit im Sinne des $\S 40$ Abs. 1 S. 1 VwGO angenommen. Ob eine Streitigkeit öffentlich-rechtlich oder zivilrechtlich ist, richtet sich nach der Natur des Rechtsverhältnisses, aus dem der geltend gemachte Anspruch hergeleitet wird. Dabei kommt es regelmäßig darauf an, ob die Beteiligten zueinander in einem hoheitlichen Verhältnis der Über- und Unterordnung stehen und sich der Träger hoheitlicher Gewalt der besonderen Rechtssätze des öffentlichen Rechts bedient (vgl. BVerwG, Beschluss v. 2. Mai 2007 - 6 B 10.07, [...] ). Es ist allgemein anerkannt, das die Unterlassung und der Widerruf von Äußerungen, die von einer staatlichen Stelle in dienstlicher Eigenschaft abgegeben werden, im Verwaltungsrechtsweg geltend zu machen sind; (vgl. Kopp/Schenke, VwGO, 19. Aufl. 2013, § 40 Rn. 28 mwN; Sodan/Ziekow-Sodan, VwGO, 3. Aufl. 2010, § 40 Rdnr. $421 \mathrm{mwN}$ ). Die Koordinierungsstelle A-Stadt ist sachlich, personell und haushaltsrechtlich dem Kultusministerium des Landes Sachsen-Anhalt als unselbständige Organisationseinheit in Gestalt einer Arbeitsgruppe zugeordnet. Bereits aus dem Wortlaut der „Erklärung der Bundesregierung, der Länder und der kommunalen Spitzenverbände zur Auffindung und zur Rückgabe NS-verfolgungsbedingt entzogenen Kulturgutes, insbesondere aus jüdischem Besitz" vom 9. Dezember 1999, welche zur Einrichtung der Lost Art Datenbank führte, ist zu entnehmen, dass die Hilfe bei der Rückführung der sogenannten Raubkunst entgegen der Auffassung des Beklagten nicht als bloße Wahrnehmung einer moralischen Aufgabe, sondern als staatliche Aufgabe begriffen worden ist, welche nicht durch eine privatrechtlich organisierte, sondern durch eine öffentliche Einrichtung betrieben wird, welche sich bei ihrer Tätigkeit (bis auf den Vertrieb gedruckter Publikationen) keiner zivilrechtlichen Handlungsformen bedient.

Zwar ist der Einwand des Beklagten zutreffend, dass das beklagte Kultusministerium bzw. die ihm als Arbeitsgruppe angeschlossene Koordinierungsstelle nicht Beklagter hinsichtlich der erhobenen allgemeinen Leistungsklage sein kann, da das sogenannte Behördenprinzip für diese Klageart nicht gilt. Dies führt allerdings nicht zur Begründetheit der Berufung, vielmehr muss dem in entsprechender Anwendung des $\S 78$ Abs. 1 Nr. 1 Hs. 2 VwGO durch eine Rubrumsberichtigung Rechnung getragen werden, die von Amts wegen im Rechtsmittelverfahren statthaft ist, selbst wenn die fälschlich als Beklagter bezeichnete Behörde in der Vorinstanz als Beklagte behandelt worden ist (vgl. BVerwG, Urteil v. 3. März 1989 - 8 C 98.85, [...]). In einer solchen Berichtigung des Passivrubrums liegt kein Austausch von Beteiligten; es wird damit nur klargestellt, dass die Behörde, die für die in Anspruch genommene Körperschaft tätig geworden ist, als deren Vertreterin am Verfahren beteiligt ist, nicht aber selbst die Rechtsstellung eines Beteiligten hat (vgl. OVG Lüneburg, Beschluss v. 4. Juli 2007 - 5 ME 131/07, [...] mwN). Dementsprechend ist die Klage gegen das Land Sachsen-Anhalt, vertreten durch den Kultusminister zu richten und das Rubrum entsprechend zu berichtigen. 
Die Klage ist entgegen der Auffassung des beklagten Landes auch nicht gegen die Koordinierungsstelle zu richten, weil die Koordinierungsstelle weder eine juristische Person öffentlichen Rechts noch eine Behörde ist. Vielmehr handelt es sich um eine unselbständige Untergliederung des Kultusministeriums. Das folgt aus § 1 Abs. 1 der Gemeinsamen Vereinbarung über die Koordinierungsstelle A-Stadt 2010-2016 vom 10. November 2009, wonach die Koordinierungsstelle eine von Bund und Ländern getragene Einrichtung in Form „einer Arbeitsgruppe des Kultusministeriums des Landes Sachsen-Anhalt in A-Stadt" ist.

Der Kläger verfolgt sein Begehren auf Löschung des Interneteintrages zu Recht im Wege der allgemeinen Leistungsklage und nicht der - ein Vorverfahren voraussetzenden - Verpflichtungsklage. Der Beklagte hat die Eintragung im Wege des Realakts veranlasst, sodass auch deren Beseitigung als "actus contrarius" lediglich einen Realakt voraussetzt.

Die Klägerin ist entgegen der Auffassung der Beigeladenen auch klagebefugt. Allein der Umstand, dass die Beigeladenen nachhaltig bestreiten, dass das streitgegenständliche Gemälde jemals im Eigentum der Galerie F. stand, führt nicht zur Verneinung der Klagebefugnis. Für die Klagebefugnis nach § 42 Abs. 2 VwGO erforderlich aber auch ausreichend ist, dass ein Kläger Tatsachen vorträgt, die es denkbar und möglich erscheinen lassen, dass er in einer eigenen rechtlichen Position beeinträchtigt ist (vgl. BVerwG, Urteil v. 27. November 1996 - 11 A 100.95, [...]). Es ist in Anlegung dieses Maßstabes nicht ausgeschlossen, dass sich die Klägerin auf eine Beeinträchtigung der Rechte aus Art. 14 Abs. 1 GG und Art. 2 Abs. 1 GG iVm Art. 19 Abs. 3 GG berufen kann. „Eigentum“ iSd Art. 14 GG und Art. 1 Zusatzprotokoll zur EMRK erfasst dabei nicht nur "Vorhandenes Eigentum", sondern kann auch Forderungen umfassen (vgl. BVerfG, Beschluss v. 14. Juli 1999 - 1 BvR 995/95 u.a., [...]; EGMR, Entscheidung v. 8. Dezember 2011, Az. 71916/01 u.a., [...]). Art. 2 Abs. 1 GG enthält das Grundrecht des Bürgers, nur auf Grund solcher Vorschriften bzw. solcher staatlicher Handlungen mit einem Nachteil belastet zu werden, die formal und materiell verfassungsgemäß sind (vgl. BVerwG, Beschluss v. 19. Juli 2010 - 6 B 20.10, [...]). Da nach der Art der geschützten Tätigkeit nicht differenziert wird, sind von Art. 2 Abs. 1 GG auch wirtschaftliche Handlungen erfasst. Geschützt werden natürliche und juristische Personen sowie Personenmehrheiten (vgl. BVerfG, Beschluss v. 19. Dezember 1967 - 2 BvL 4/65, [...]).

Entgegen der Auffassung des Beklagten fehlt der Klage auch nicht das erforderliche Rechtsschutzbedürfnis. Ein Rechtsschutzbedürfnis ist zu verneinen, wenn der Rechtsschutzsuchende sein Ziel sachgerechter - insbesondere einfacher, umfassender, schneller oder billiger - erreichen kann. Dies ist vor allem dann der Fall, wenn ein Kläger eine rechtsschutzintensivere Rechtsschutzform wählen konnte (Schoch/Schneider/ Bier-Ehlers, VwGO, Vorbemerkung $\S 40$ Rn. 81). Entgegen der Auffassung des Beklagten kann die Klägerin nicht darauf verwiesen werden, zunächst in einem zivilrechtlichen Verfahren gegenüber den Beigeladenen zu klären, wer Eigentümer an dem streitgegenständlichen Gemälde ist bzw. als Berechtigter an dem Gemälde gilt. Das Verwaltungsgericht hat zutreffend darauf hingewiesen, dass bereits nicht alle Angehörigen der Erbengemeinschaften nach den vormaligen Gesellschaftern des Bankhauses Jacquier \& Securius bekannt sind, was jedenfalls eine erhebliche Erschwerung einer zivilgerichtlichen Rechtsverfolgung bedeuten würde. Hinzu kommt, dass ein solcher Rechtsstreit nicht notwendigerweise vor einem deutschen Gericht zu führen wäre. Mangels materieller Rechtsgrundlage für den Betrieb der Lost Art Datenbank sähe sich die Klägerin hierbei dem Risiko ausgesetzt, dass auch nach Klärung der Eigentumsfrage zu ihren Gunsten der Beklagte die Löschung der Suchmeldung etwa unter Hinweis auf eine fehlende Validität einer ausländischen gerichtlichen Entscheidung ablehnen könnte und gleichwohl die Durchführung eines verwaltungsgerichtlichen Verfahrens erforderlich wäre.

Das Verwaltungsgericht hat den Beklagten zu Recht verurteilt, die Suchmeldung zu löschen.

Für die von der Klägerin begehrte Löschung der Suchmeldung kommt als Anspruchsgrundlage nur der gesetzlich nicht geregelte, jedoch gewohnheitsrechtlich anerkannte und durch Richterrecht geprägte öffentlich-rechtliche Folgenbeseitigungsanspruch in Betracht. Der Folgenbeseitigungsanspruch entsteht, wenn durch einen hoheitlichen Eingriff in ein subjektives Recht ein noch andauernder rechtswidriger Zustand geschaffen worden ist; er ist auf die Wiederherstellung des Zustands gerichtet, der im Zeitpunkt des Eingriffs bestand. Der Folgenbeseitigungsanspruch knüpft mithin nicht an die Rechtswidrigkeit des Eingriffsakts, sondern an die Rechtswidrigkeit des dadurch geschaffenen Zustands an. Ihm liegt die sowohl grundrechtlich als auch rechtsstaatlich motivierte Forderung zugrunde, diesen Zustand mit der rechtsnormativen Lage zur Deckung zu bringen (vgl. BVerwG, Urteil v. 23. Mai 1989 - 7 C 2.87, [...]). Entscheidend ist dabei, ob die beeinträchtigende Einrichtung in einem öffentlich-rechtlichen Planungs- und Funktionszusammenhang steht. Ein solcher Planungs- und Funktionszusammenhang ist gegeben, wenn - wie hier - die betreffende Einrichtung der Öffentlichkeit gewidmet ist und öffentlichen Zwecken dient (vgl. OVG Lüneburg, Urteil v. 31. März 2004 - 13 LB $11 / 03,[. ..] \mathrm{mwN})$.

Gemessen an diesen Maßstäben hat die Klägerin im Rahmen des Folgenbeseitigungsanspruches einen Anspruch auf Löschung der Suchmeldung hinsichtlich des streitgegenständlichen Gemäldes, da der Zweck der Eintragung der Suchmeldung in die Lost Art Datenbank mit dem Auffinden des Bildes in Südafrika im Jahr 2009 erfüllt ist, die Eigentumsprätendenten Gelegenheit hatten, die von ihnen geltend gemachten Ansprüche an dem Gemälde zu sichern und ein Fortbestand der Eintragung die Klägerin in ihren rechtlichen geschützten Interessen verletzt. 
Die Rechtmäßigkeit eines Eintrages in der Lost Art Datenbank ist nach den für den Bereich der staatlichen - nicht regelnden - Informationstätigkeit entwickelten Maßstäben zu beurteilen. Obwohl es sich bei diesem Informationshandeln - abgesehen von amtlichen Warnungen - regelmäßig nicht um eine final eingreifend tätige wirtschaftsverwaltungsrechtliche Aufsicht des Staates handelt, ist eine Grundrechtsrelevanz einer solchen Tätigkeit nicht generell zu verneinen. Eine beeinträchtigende Wirkung des Grundrechtsträgers ist nicht unmittelbar auf eine staatliche Maßnahme (z.B. ein Verkaufsverbot oder eine Geschäftsschließung), sondern nur mittelbar auf die Reaktion von Marktteilnehmern auf die staatliche Information zurückzuführen (vgl. Becker/Blackstein, NJW 2011, 490 zur staatlichen Verbraucherinformation über das Internet; Schoch, NJW 2012, 2844 zur Verbraucherinformation im Lebensmittel-, Produktsicherheits- und Sozialversicherungsrecht). Entscheidend für die Erforderlichkeit einer Ermächtigungsgrundlage ist dabei, ob es sich bei staatlichen Verbraucherinformationen um Eingriffe in verfassungsrechtlich geschützte Interessen z.B. in die Berufsfreiheit, das Recht auf Eigentum oder das allgemeine Persönlichkeitsrecht handelt. Bereits der Eingriffscharakter und nicht erst die verfassungsrechtliche Rechtfertigung einer staatlichen Informationsmaßnahme hängt nach der Rechtsprechung des Bundesverfassungsgerichts davon ab, ob die staatliche Informationstätigkeit in Erfüllung einer zugewiesenen staatlichen Aufgabe erfolgt, die Zuständigkeitsordnung eingehalten worden ist und die weitergegebenen Informationen richtig und sachlich sind (vgl. BVerfG, Beschlüsse v. 26. Juni 2002 - 1 BvR 670/91, [...] und - 1 BvR 558/91, - 1 BvR 1428/91, [...]). Darüber hinaus muss das staatliche Informationshandeln ein legitimes Ziel verfolgen und sich gemessen daran als verhältnismäßig erweisen (vgl. BVerfG, Beschluss v. 17. August 2010 - 1 BvR 2585/06, [...] zu den Anforderungen an Stellungnahmen der Bundeszentrale für politische Bildung; OVG Münster, Urteil v. 17. September 2013 13 A 2541/12, [...] zu behördlichen Warnungen vor sogenannten E-Zigaretten). Können Aufgaben der Regierung oder der Verwaltung mittels öffentlicher Informationen wahrgenommen werden, liegt nach Auffassung des Bundesverfassungsgerichts in der Aufgabenzuweisung grundsätzlich auch eine Ermächtigung zum Informationshandeln. Insbesondere könne die staatliche Informationstätigkeit eine Beeinträchtigung im Gewährleistungsbereich des Grundrechts sein, wenn sie in der Zielsetzung und ihren Wirkungen Ersatz für eine staatliche Maßnahme ist, die als Grundrechtseingriff zu qualifizieren wäre. Durch Wahl eines solchen funktionalen Äquivalents eines Eingriffs können die besonderen Bindungen der Rechtsordnung nicht umgangen werden; vielmehr müssen die für Grundrechtseingriffe maßgebenden rechtlichen Anforderungen erfüllt sein. Ebenfalls wird der Gewährleistungsbereich beeinträchtigt, wenn eine Information sich im Nachhinein als unrichtig erweist und dennoch weiterverbreitet oder nicht korrigiert wird, obwohl sie für das Marktverhalten weiter von Belang ist. Mit der Feststellung der Beeinträchtigung des Schutzbereichs steht in solchen Fällen auch die Rechtswidrigkeit fest, da eine Rechtfertigung der Weiterverbreitung der als unrichtig erkannten Information ausgeschlossen ist (BVerfG, Beschluss v. 26. Juni 2002 - 1 BvR 558/91, - 1 BvR 1428/91, aaO).

Der Senat lässt es offen, ob gemessen an diesen Maßstäben der Betrieb der Lost Art Datenbank einer gesetzlichen Ermächtigung bedarf. Die Aufrechterhaltung der von der Klägerin im Jahr 2005 veranlassten Suchmeldung hinsichtlich des streitgegenständlichen Gemäldes ist jedenfalls deshalb rechtswidrig, da der Zweck der Eintragung in der Suchliste der Lost Art Datenbank erfüllt war.

Entgegen der Auffassung der Beigeladenen erfüllt die Suchliste der Lost Art Datenbank nicht den Zweck, allgemein das Wiedergutmachungsinteresse natürlicher oder juristischer Personen an sogenannter Raubkunst zu dokumentieren, was aus Sicht der Beigeladenen bedeutet, dass eine Löschung unabhängig vom Willen der Anmelder erst erfolgen kann, wenn die eigentumsrechtliche Zuordnung eines der sogenannten Raubkunst zugeordneten Kunstgegenstandes geklärt ist.

Da die Einrichtung und der Betrieb der Datenbank nicht in materiellen Rechtsvorschriften geregelt ist, können zur Bestimmung des Zweckes der in der Datenbank enthaltenen Suchliste nur die vom Träger bzw. Trägern der öffentlichen Einrichtung hierzu abgegebenen Willenserklärungen in Betracht kommen. Am 3. Dezember 1998 wurde im Anschluss an die "Washington Conference on Holocaust-Era Assets", an der 44 Staaten, darunter auch die Bundesrepublik Deutschland, sowie eine Reihe nichtstaatlicher Organisationen teilnahmen, die so genannte „Washingtoner Erklärung" mit elf Leitsätzen veröffentlicht (Materialien zur Konferenz veröffentlicht unter: fcit.usf.edu/HOLOCAUST/RESOURCE/assets/index.htm; deutsche (nichtamtliche) Übersetzung der sogenannten Washington Principles bei Hartung, Kunstraub in Krieg und Verfolgung, 2005, S. 105 f.). In dieser Erklärung verpflichteten sich die Konferenzteilnehmer, Kunstwerke, die während der Zeit des Nationalsozialismus beschlagnahmt wurden, ausfindig zu machen, die rechtmäßigen Eigentümer oder deren Erben zu finden und rasch die notwendigen Schritte zu unternehmen, um zu "fairen und gerechten“ Lösungen zu gelangen. Diese Erklärung enthält weder eine rechtlich bindende Verpflichtung, noch begründet sie (neue) Individualrückgabeansprüche von Betroffenen, wie sich bereits aus dem Eingangssatz der Erklärung ergibt („In developing a consensus on non-binding principles to assist in resolving issues relating to Nazi-confiscated art, the Conference recognizes that among participating nations there are differing legal systems and that countries act within the context of their own laws", so auch BVerwG, Urteil v. 24. November 2011 - 7 C 12.10, [...]). Es wurde vielmehr darauf hingewiesen, dass die beteiligten Staaten Mechanismen der außergerichtlichen Streitbelegung zur Klärung von streitigen Eigentumsfragen nutzen sollten. Im Anschluss an die Washingtoner Konferenz haben die Bundesregierung, die Länder und die kommunalen Spitzenverbände am 9. Dezember 1999 eine gemeinsame Erklärung veröffentlicht. Dort heißt es unter Ziffer III.: 
„Darüber hinaus prüfen Bundesregierung, Länder und kommunale Spitzenverbände im Sinne der Washingtoner Grundsätze ein Internet-Angebot einzurichten, das folgende Bereiche umfassen sollte:

1. Möglichkeiten der beteiligten Einrichtungen, Kulturgüter ungeklärter Herkunft zu veröffentlichen, sofern NS-verfolgungsbedingter Entzug vermutet wird.

2. Eine Suchliste, in die jeder Berechtigte die von ihm gesuchten Kulturgüter eintragen und damit zur Nachforschung für die in Frage kommenden Einrichtungen und die interessierte Öffentlichkeit ausschreiben kann.

3. Informationen über kriegsbedingte Verbringung NS-verfolgungsbedingt entzogener Kulturgüter in das Ausland.

4. Die Schaffung eines virtuellen Informationsforums, in dem die beteiligten öffentlichen Einrichtungen und auch Dritte ihre Erkenntnisse bei der Suche nach NS-verfolgungsbedingt entzogenen Kulturgütern eingeben können, um Parallelarbeiten zu gleichen Themen (z.B.: Bei welcher Auktion wurden jüdische Kulturgüter welcher Sammlung versteigert?) auszuschließen und im Wege der Volltextrecherche schnell zugänglich zu machen."

Um das Wissen über Kulturgutverluste zu dokumentieren, die Verluste der deutschen Institutionen zu erfassen und somit eine Grundlage für die Suche und Rückführung dieser Kulturgüter zu schaffen, hatten die Länder Berlin, Brandenburg, Bremen, Hamburg, Mecklenburg-Vorpommern, Niedersachsen, Sachsen, Sachsen-Anhalt, Schleswig-Holstein und Thüringen bereits 1994 in Bremen die Koordinierungsstelle der Länder für die Rückführung von Kulturgütern gegründet. Seit 1998 beteiligen sich alle 16 Länder an der Koordinierungsstelle mit Sitz in A-Stadt. Im Zusammenhang mit der durch die vorgenannte Gemeinsame Erklärung eingetretenen Aufgabenerweiterung ging die bisherige Koordinierungsstelle im Januar 2001 in der Koordinierungsstelle A-Stadt als gemeinsame Einrichtung aller Länder und des Bundes auf. Finanzierung und Organisation der Koordinierungsstelle sind in einer zeitlich befristet geschlossenen Verwaltungsvereinbarung zwischen dem Bund und den Ländern geregelt (Gemeinsame Vereinbarung über die Koordinierungsstelle A-Stadt 2010-2016). Zu den Aufgaben der Koordinierungsstelle heißt es unter $\S 1$ Abs. 3 Buchst. a und b der Verwaltungsvereinbarung: „Die Koordinierungsstelle hat die folgenden Aufgaben: a. Dokumentation von Such- und Fundmeldungen des In- und Auslands zu NS-verfolgungsbedingt entzogenen bzw. infolge des Zweiten Weltkriegs verbrachten Kulturgütern zur Präsentation in www.lostart.de, b. Gewährleistung der Funktionsfähigkeit und kontinuierliche Überarbeitung des Angebotes von Datenbank und Website mit dem Ziel des weiteren Ausbaus zu einem Informationsportal (einschl. Forum)." Inhaltlich orientiert sich die Koordinierungsstelle auch an der "Handreichung zur Umsetzung der Erklärung der Bundesregierung, der Länder und der kommunalen Spitzenverbände zur Auffindung und zur Rückgabe NS-verfolgungsbedingt entzogenen Kulturgutes, insbesondere aus jüdischem Besitz vom Dezember 1999" vom Februar 2001, überarbeitet im November 2007, welche unter Leitung des Beauftragten der Bundesregierung für Kultur und Medien erstellt worden ist. Auch wenn in dieser Handreichung hinsichtlich der Prüfung von Herausgabeverlangen auf das alliierte Rückerstattungsrecht und das Vermögensgesetz verwiesen wird, wird an mehreren Stellen ausdrücklich betont, dass es sich bei der Handreichung nicht um ein verbindliches rechtliches Regelwerk handelt, sondern lediglich um die Anregung, bei der Prüfung des Herausgabeverlangens den Leitlinien der rückerstattungsrechtlichen Praxis der Nachkriegszeit zu folgen (Seite 27 der Handreichung).

Wie sich aus den vorgenannten Unterlagen ergibt, ist die der Lost Art Datenbank zugewiesene Funktion daher beschränkt auf die Veröffentlichung von Such- und Fundmeldungen hinsichtlich solcher unrechtmäßig den Eigentümern entzogenen Kulturgüter, welche von der Washingtoner Erklärung und der Gemeinsamen Erklärung des Bundes und der Länder von 1999 erfasst werden. In der Suchliste der Datenbank sind die Kulturgüter verzeichnet, die öffentlichen Einrichtungen oder privaten Personen und Institutionen infolge der nationalsozialistischen Gewaltherrschaft und des Zweiten Weltkrieges als verloren gegangen gemeldet haben und über die Internet-Datenbank zur weltweiten Suche ausgeschrieben wurden. Besitzer oder Verwalter von Kulturgütern mit unsicherer oder lückenhafter Provenienz sollen hier recherchieren können, ob diese anderenorts gesucht werden können. Diese Tätigkeit des Beklagten im Zusammenhang mit dem Betrieb der Lost Art Datenbank ist auch dem Bereich des staatlichen Informationshandelns zuzurechnen, da sich der Beklagte nicht nur darauf beschränkt, ohne jegliche eigene Wertung und ungeprüft Suchmeldungen Dritter im Internet zu veröffentlichen. Der Beklagte nimmt vielmehr vor der Eintragung einer Suchmeldung eine Plausibilitätsprüfung zumindest zur Frage vor, ob es sich bei dem Kulturgut um ein solches handeln kann, welches zwischen 1933 und 1945 den damaligen Eigentümern aufgrund von Verfolgungsmaßnahmen zu Unrecht entzogen worden ist („Grundsätze zur Eintragung und zur Löschung von Meldungen zu Kulturgütern in www.lostart.de" sowie "Checkliste Plausibilitätsprüfung", Stand Mai 2013, jeweils veröffentlicht unter www.lostart.de). Es heißt in diesen Grundsätzen ausdrücklich, dass im Rahmen dieser Plausibilitätsprüfung geprüft werde, ob die übermittelten Informationen dem Grunde nach die Berechtigung zur Eintragung nachvollziehbar darlegen und insgesamt keine offenkundigen Widersprüche erkennen lassen. Sollten die vom Melder übermittelten Angaben der Plausibilitätsprüfung nicht standhalten, behält sich der Beklagte vor, diese Meldung nicht zu veröffentlichen. Entgegen der Auffassung des Beklagten handelt es sich bei der Eintragung dieser Such- und Fundmeldungen nicht nur um eine bloße deskriptive Tätigkeit, bei der der Beklagte ohne eigene Wertung etwa im Sinne eines Ausstellungskataloges lediglich Beschreibungen Dritter von Kunstwerken aufnimmt. Die Lost Art Datenbank unterscheidet sich daher auch von staatlich eingerichteten bzw. finanzierten Internetportalen, auf denen 
in aggregierter Form Private ihre Bewertungen hinsichtlich bestimmter Lebenssachverhalte einstellen können und sich die staatlichen Stellen auf die bloße Verbreitung der subjektiven Einschätzungen privater Dritter beschränken (zum sogenannten kollaborativen Informationshandeln: Martini/Kühl, DÖV 2013, 573 f. hinsichtlich sogenannter Bewertungsportale im Bereich des Sozialversicherungs- und Lebensmittelrechts).

Dieser Zweck der Suchliste ist mit dem Auffinden des Bildes bei dem Besitzer erfüllt. Insofern kann sich der Beklagte auch nicht darauf berufen, dass die Beigeladenen einer Löschung der Suchmeldung (noch) nicht zugestimmt haben. Der Verbleib der Suchmeldung in der Datenbank hat sich allein danach zu beurteilen, ob die der öffentlichen Einrichtung zugewiesene Aufgabe noch zu erfüllen ist.

Auch der Einwand des Beklagten, dass durch einen Disclaimer (Haftungsausschluss) auf der Homepage klargestellt sei, dass keine Verantwortung für die inhaltliche Richtigkeit der von Dritten übermittelten Daten übernommen werde und er daher nicht richtiger Adressat des Begehrens der Klägerin sei, mag im Hinblick auf eine strafrechtliche Verantwortung oder Schadensersatzansprüche von Bedeutung sein. Wie sich aus der Aufmachung der Homepage und insbesondere der vom Beklagten auf der Homepage veröffentlichten Checkliste zur Plausibilitätsprüfung ergibt, stellen die veröffentlichten Suchmeldungen jedoch keinen sogenannten ausschließlichen Fremdinhalt dar, der dem Beklagten als Betreiber der Homepage nicht zurechenbar wäre.

Entgegen der Auffassung der Beigeladenen erfüllt die Datenbank auch keine weiterreichende anspruchssichernde Funktion in dem Sinne, dass die Suchmeldung so lange aufrechtzuerhalten ist, solange die eigentumsrechtliche Berechtigung hinsichtlich des streitgegenständlichen Gemäldes nicht (etwa durch ein rechtskräftiges Urteil eines deutschen Gerichts) geklärt ist. Eine solche Funktion der Datenbank ergibt sich weder aus der Washingtoner Erklärung, der gemeinsamen Erklärung von 1999, der Verwaltungsvereinbarung des Bundes und der Länder noch der Handreichung des Beauftragten des Bundes für Kultur und Medien. Die Lost Art Datenbank erfüllt als Suchund Funddatenbank daher eine andere Funktion als etwa $\S 30 \mathrm{~b}$ VermG in der seit dem 9. Oktober 2013 geltenden Fassung (Gesetz v. 1. Oktober 2013, BGBI. I S. 3719, 3727), wonach bei Grundstücken, für welche eine vermögensrechtliche Anmeldung vorliegt, über welche noch nicht bestandskräftig entschieden worden ist, von Amts wegen ein sogenannter Anmeldevermerk in die Abteilung II des Grundbuches einzutragen ist. Der Beklagte weist auf seiner Internetseite selbst darauf hin, dass im Falle eines Prätendentenstreites es Aufgabe der Beteiligten sei, zur Sicherung von Ansprüchen z.B. eine Hinterlegung oder eine sonstige Sicherungsmaßnahme zu bewirken.

Die nicht mehr vom Zweck der Suchliste umfasste Aufrechterhaltung der Eintragung des streitgegenständlichen Gemäldes führt auch zu einer Rechtsverletzung der Klägerin.
Soweit das Verwaltungsgericht hierzu selbständig tragend ausführt, dass die Klägerin zwar das Eigentum an dem streitgegenständlichen Gemälde verfolgungsbedingt verloren habe, sie jedoch in entsprechender Anwendung des $\S 3$ Abs. 2 VermG als sogenannte Erstgeschädigte im Hinblick auf eine vermögensrechtliche Berechtigung einen auf Löschung der Eintragung gerichteten Abwehranspruch gegenüber dem Beklagten geltend machen könne, ist zunächst darauf zu verweisen, dass es keine materiell-rechtliche Rechtsgrundlage gibt, welche hinsichtlich der Prüfung von „berechtigten“ Eintragungen in die Suchliste der Lost Art Datenbank durch den Beklagten die Regelungen des Vermögensgesetzes für anwendbar erklärt. Auch wenn formal der Anwendungsbereich des Vermögensgesetzes ( $§$ 6 VermG) nicht ausgeschlossen ist, da sowohl die F. \& Co. GmbH (zeitweilig) als auch das Bankhaus Jacquier \& Securius zwischen 1933 und 1945 ihren Sitz im später sowjetisch besetzten Teil von Berlin hatten, ist der Anwendungsbereich des Vermögensgesetzes grundsätzlich nur (noch) hinsichtlich solcher Rückübertragungsverfahren eröffnet, welche noch nicht rechtskräftig abgeschlossen sind. Hierfür sind hinsichtlich des streitgegenständlichen Gemäldes keine Anhaltspunkte ersichtlich (zum Entschädigungsverfahren hinsichtlich des Bankhauses Jacquier \& Securius: VG Berlin, Urteil v. 27. September 2012 - 29 K 269.10, [...]). Zudem ist nicht ersichtlich, dass es sich bei der Prioritätsregel in $\S 3$ Abs. 2 VermG um einen allgemeinen Grundsatz des Rückerstattungsrechts handelt (vgl. zum Gesetzeszweck: BVerwG, Beschluss v. 29. Dezember 2010 - 8 B 31.10, [...]). Das Rückerstattungsrecht in der US-amerikanischen Zone und in der britischen Zone, an welches partiell auch das Vermögensgesetz in $\S 1$ Abs. 6 VermG anknüpft, ist der Sache nach eine besondere Materie des Zivilrechts (vgl. Rudolph, Restitution von Kunstwerken aus jüdischem Besitz, 2007, S. 85 f.; Anton, Illegaler Kulturgüterverkehr, 2010, S. 687, jeweils mwN), welches insbesondere keine staatlichen Entschädigungsleistungen für einen zeitlich nachrangig Geschädigten vorsah. Soweit die Klägerin davon ausgeht, dass sie das Eigentum an dem Gemälde nicht verloren habe, wäre ungeachtet der Frage, ob nach dem Ablauf der Ausschlussfristen nach dem Rückerstattungsrecht noch zivilrechtliche Herausgabeansprüche geltend gemacht werden können (vgl. BGH, Urteil v. 16. März 2012 - V ZR 279/10, [...] „Plakatsammlung Sachs"), bei einer zivilrechtlichen Betrachtungsweise - da das Gemälde sich derzeit nicht in der Bundesrepublik Deutschland befindet - zur Bestimmung des anwendbaren Rechts die Anknüpfungsregeln des (deutschen) internationalen Sachenrechts zu beachten, wonach zumindest nicht ausgeschlossen werden kann, dass nicht deutsches Sachenrecht zur Beurteilung der Eigentumsfrage heranzuziehen ist (vgl. hierzu Kiechle, NJOZ 2011, 193 mwN). Insofern ist es auch nicht ausgeschlossen, dass nach zivilrechtlichen Regelungen durch gutgläubigen Erwerb, Ersitzung oder vergleichbare zivilrechtliche Erwerbstatbestände nach 1945 ein Dritter - möglicherweise rechtlich anfechtbares - Eigentum an dem streitgegenständliche Gemälde erworben hat und damit - derzeit - weder die Kläger noch die Beigeladenen sich auf das Eigentum an dem Gemälde berufen können. 
Selbst wenn man mit der Klägerin davon ausgeht, dass sie das Eigentum an dem streitgegenständlichen Gemälde nicht verloren habe, kann sie sich nicht auf eine Verletzung eines durch Art. 14 Abs. 1 GG geschützten Rechts berufen. Art. 14 Abs. 1 GG gewährleistet zwar das Recht, Sach- und Geldeigentum zu besitzen, zu nutzen, es zu verwalten und darüber zu verfügen. Eine allgemeine Wertgarantie vermögenswerter Rechtspositionen kann aus dieser Vorschrift allerdings nicht abgeleitet werden. Der Tauschwert vermögenswerter Rechte unterfällt für sich genommen nicht dem Schutzbereich der Eigentumsfreiheit. Hoheitlich bewirkte Minderungen des Tausch- oder Marktwertes eines Eigentumsgutes berühren daher in der Regel nicht das Eigentumsgrundrecht (vgl. BVerfG, Urteil v. 11. Juli 2012 - 1 BvR 3142/07, - 1 BvR 1569/08, [...]; Beschluss v. 5. Februar 2002 - 2 BvR 305/93, - 2 BvR 348/93, [...], jeweils mwN). Insoweit wäre mit der von der Klägerin vorgetragenen Beeinflussung der Verkehrsfähigkeit des Gemäldes durch die Aufrechterhaltung der Suchmeldung in der Lost Art Datenbank selbst dann kein Eingriff in ein nach Art. 14 GG geschütztes Recht verbunden, wenn man die eigentumsrechtliche Stellung der Klägerin an dem Gemälde bejahen würde.

Die Rechtswidrigkeit der weiteren Aufrechterhaltung der Registrierung der Suchmeldung führt jedoch zur Verletzung der allgemeinen Handlungsfreiheit der Klägerin nach Art. 2 Abs. 1 GG.

Art. 2 Abs. 1 GG schützt die freie Entfaltung der Persönlichkeit. Sie umfasst neben dem allgemeinen Persönlichkeitsrecht auch die allgemeine Handlungsfreiheit. Teil dieser umfassenden Garantie, die jede menschliche Betätigung einschließt, welche nicht den Schutz eines speziellen Grundrechts genießt, ist auch die wirtschaftliche Betätigungsfreiheit (vgl. BVerfG, Beschluss v. 8. April 1997 - 1 BvR 48/94, [...]). Die wirtschaftliche Handlungsfreiheit wird durch Maßnahmen betroffen, die auf Beschränkung wirtschaftlicher Entfaltung sowie Gestaltung, Ordnung oder auch Lenkung des Wirtschaftslebens angelegt sind oder sich in diesem Sinne auswirken (vgl. BVerfG, Urteil v. 14. Juli 1998 - 1 BvR 1640/97, [...]). Diese wirtschaftliche Handlungsfreiheit ist nur in den durch das Grundgesetz bestimmten Schranken garantiert, vor allem denen der verfassungsmäßigen Ordnung (vgl. BVerfG, Beschluss v. 16. Juli 2012 - 1 BvR 2983/10, [...]). Die wirtschaftliche Handlungsfreiheit enthält die Gewährleistung, nur auf Grund solcher Vorschriften mit einem Nachteil belastet zu werden, die formal und materiell der Verfassung gemäß sind (vgl. BVerfG, Beschluss v. 12. April 2005 - 2 BvR 1027/02, [...]; BVerwG, Beschluss v. 19. Juli 2010 - 6 B 20.10, [...]).

Durch die Aufnahme der Suchmeldung in die Lost Art Datenbank wird einem Kulturgut durch den Beklagten ein bestimmtes (wertbildendes und wertbestimmendes) Attribut zugeordnet, nämlich dass bei diesem Kulturgut zumindest der Verdacht besteht, dass es sich um sogenannte Raubkunst handelt. Es heißt hierzu auf der Internetseite zur Datenbank ausdrücklich: „Die Lost Art Internet-Datenbank enthält Angaben zu Kulturgütern, die infolge des Nationalsozialismus bzw. des Zweiten Weltkrie- ges verbracht, verlagert oder insbesondere jüdischen Eigentümern verfolgungsbedingt entzogen wurden oder für die auf Grund von Provenienzlücken eine solche Verlustgeschichte nicht ausgeschlossen werden kann." Wie sich aus dem insofern übereinstimmenden Vortrag der Klägerin und der Beigeladenen ergibt, hat eine Suchmeldung in der Lost Art Datenbank im Internet (wie auch in einer der in Großbritannien ansässigen vergleichbaren Datenbanken Art Loss Register und lootedart. com) für die Verkehrsfähigkeit eines Kunstgegenstandes insofern eine erhebliche Bedeutung, als dieses Werk mit dem „Makel" behaftet ist, dass zumindest der Verdacht besteht, dass es sich um sogenannte Raubkunst handelt. Veräußerer bzw. Erwerber eines in der allgemein zugänglichen Suchliste der Lost Art Datenbank aufgeführten Kunstgegenstandes müssen in Betracht ziehen, dass hinsichtlich dieses Werkes möglicherweise nicht wirksam Eigentum erworben werden kann bzw. das Eigentum mit einem Rückübertragungsanspruch belastet ist. Auch wenn es sich bei der Suchliste der Lost Art Datenbank weltweit gesehen nicht um die einzige Informationsquelle zu sogenannter Raubkunst handelt und Kunsthändler bzw. Auktionshäuser rechtlich nicht verpflichtet sind, sich vor einer Veräußerung bzw. Versteigerung durch eine Recherche in der Lost Art Datenbank zu vergewissern, ob ein bestimmtes Kunstwerk dort in der Suchliste registriert ist, handelt es sich bei dieser Datenbank gleichwohl um ein wichtiges Informationsmedium zum Auffinden von sogenannter Raubkunst. So werden nach den Angaben des Beklagten monatlich 1,6 Millionen Zugriffe auf das Portal "lostart.de" registriert (Spiegel-Online v. 31. Januar 2013 „Jäger der verlorenen Kunstschätze“). Nach Überzeugung des Senates belegen diese hohen Zugriffszahlen, dass die in der Suchliste vom Beklagten aufgenommenen Informationen zur sogenannten Raubkunst für den nationalen und internationalen Kunsthandel von hoher Bedeutung sind. Der in der Suchliste der Lost Art Datenbank öffentlich dokumentierte Makel eines Kunstgegenstandes, dass er zumindest mit dem Verdacht behaftet ist, seinen Eigentümern aufgrund nationalsozialistischer Verfolgungsmaßnahmen zwischen 1933 und 1945 zu Unrecht entzogen worden zu sein, führt nicht nur zu einem merkantilen Minderwert, sondern kann im Einzelfall zur zeitweiligen Unveräußerlichkeit des Werkes führen, wie exemplarisch die von der Klägerin unter Hinweis auf die Eintragung des streitgegenständlichen Gemäldes in der Lost Art Datenbank (und Art Loss Register) angestrengte und erfolgreiche Intervention bei dem in Südafrika ansässigen Auktionshaus Rudd im Jahr 2009 belegt. Diese mit der Eintragung in die Lost Art Datenbank verbundene Beschränkung der wirtschaftlichen Handlungsfreiheit ist durch die betroffenen Grundrechtsträger nur solange zu dulden, wie es der Zweck der Suchliste, nämlich die Unterstützung bei der Suche nach verschollener Raubkunst, erfordert.

Gründe für die Zulassung der Revision (§ 132 Abs. 2 VwGO) liegen nicht vor. (Entscheidung von der Redaktion bearbeitet.) 